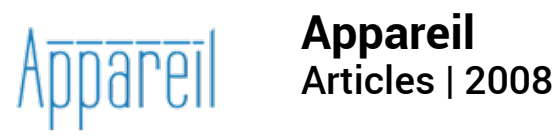

\title{
Un roi pour Israël ?
}

\section{Jean-Louis Déotte}

\section{OpenEdition \\ Journals}

\section{Édition électronique}

URL : http://journals.openedition.org/appareil/357

DOI : 10.4000/appareil.357

ISSN : 2101-0714

\section{Éditeur}

MSH Paris Nord

\section{Référence électronique}

Jean-Louis Déotte, « Un roi pour Israël ? », Appareil [En ligne], Articles, mis en ligne le 11 avril 2008 , consulté le 30 juillet 2020. URL : http://journals.openedition.org/appareil/357 ; DOI : https://doi.org/ 10.4000/appareil.357

Ce document a été généré automatiquement le 30 juillet 2020.

\section{(c) (i) (9)}

Appareil est mis à disposition selon les termes de la Licence Creative Commons Attribution - Pas d'Utilisation Commerciale - Pas de Modification 4.0 International. 


\title{
Un roi pour Israël?
}

\author{
Jean-Louis Déotte
}

\section{Introduction}

1 Ce travail de 1978, un mémoire de DEA de sociologie présenté à l'Université de Caen, est né de la rencontre entre une lecture de $\mathrm{Cl}$. Lefort et une thèse anti-étatique portant sur la société juive antique. Thèse qu'on ${ }^{1}$ étendait au judaïsme en général. Selon nous, fondamentalement, le judaïsme était la manière d'être d'une communauté qui se serait toujours pensée et constituée contre l'Etat. Que cet Etat soit le sien ou qu'il soit tel ou tel Etat "adopté" après la destruction de la nation installée en Palestine. Il convenait donc de décrire le mode d'être d'une société contre l'Etat; nous en avons trouvé le modèle dans l'étude ethnologique réalisée par Clastres des indiens Guayaki. Mais la société hébraïque n'est évidemment pas une société sauvage, pas plus d'ailleurs que la société chrétienne ou la société islamique. Ces religions de la révélation ont bien plutôt donné naissance à des nations despotiques, du type «théologico-politique ». Mais le despotisme apparaît souvent comme inessentiel, comme s'il était de la nature de ces civilisations de faire ou de tenter de faire l'économie d'une médiation terrestre entre Dieu et les hommes. De là le surgissement toujours répété des sectes, des ordres monastiques, des mystiques. Le despote et son Etat, malgré leur attachement à l'audelà, sont toujours scandaleux en ce qu'ils font surgir une nouvelle médiation grosse de finalités propres. Goitein rappelle ainsi que dans l'Islam,

« tes hommes religieux évitaient le service officiel, considérant le gouvernement en général comme l'essence même des forces qui s'opposent au règne de la loi divine sur terre. Un homme pieux n'acceptait pas une invitation à souper chez un fonctionnaire du gouvernement : la nourriture qu'on lui aurait apportée ne pouvait être considérée comme moralement halal, comme permise par ta religion: n'étaient-ce pas ses extorsions, le mépris de la loi et l'exploitation des faibles qui assuraient la plus grande part du revenu des fonctionnaires ? ${ }^{2}$

Il n'en reste pas moins que dans les trois types de religion révélée considérés le coup de force despotique a été possible, même si indéniablement celui-ci a entraîné un bouleversement dans les rapports entre l'au-delà et l'ici-bas. Il conviendrait donc de déterminer précisément, dans le cas du judaïsme, ce qu'il en était de la société pré- 
davidique afin de comprendre ce qui avait rendu possible l'émergence de la royauté. Immédiatement nous rencontrerons la question du "sujet" ou des "sujets" de ces narrations bibliques et de l'époque de celles-ci. Et d'une manière générale, si la Bible nous apporte une masse considérable de faits historiques, il est nécessaire de s'interroger sur le savoir de la société hébraïque qu'ils sont censés porter. Il est plus important de savoir qui narre que ce qui est narré, (le sens de ce qui est narré, qui relève proprement de la tâche de l'herméneute). Mais il ne s'agit pas tant d'identifier le narrateur - tâche de l'historien - que de comprendre ce que, en fonction de la nature de la société hébraïque, il ne peut que méconnaître de celle-ci. Ce qui est narré est fonction du statut de la narration. Les événements rapportés dans les écrits du scribe sont relatifs à la nature du savoir que la société a d'elle-même. Celui qui rappelle un mythe dans la société sauvage énonce bien un certain savoir sur la société, puisque celui-ci rend probablement compte d'un rituel et le fonde ainsi. Mais en ordonnant le mythe du côté des Ancêtres, le narrateur contribue - et il ne saurait y échapper - à renvoyer la société à une profonde méconnaissance d'elle-même. Cette méconnaissance est en partie du même ordre dans la société hébraïque puisque elle-même se pense en fonction d'un autre, un "sujet" divin duquel elle tient sa Loi et en fonction duquel l'autorité temporelle tient sa légitimité. Méconnaissance à laquelle pourrait bien en être liée une autre, ce qui fera toute la différence avec la société sauvage, méconnaissance qui serait celle de la division sociale. En effet, indique $\mathrm{Cl}$. Lefort :

«dans les états despotiques [...] le prince se trouve inséré dans des puissances instauratrices ou régulatrices de l'ordre du monde, car dans un tel modèle il n'y a pas à proprement parler d'ordre social distinct, autonome, visible, les hiérarchies humaines paraissent inscrites dans un univers naturel » et ajoute-t-il, «dans les états monarchiques modernes [...] tout en se réclamant de son droit divin au gouvernement des hommes, le prince renvoie à la société l'image d'une unité et d'abord d'une réalité purement sociale qui, en dépit des multiples divisions entre groupements, se donne comme un corps. ${ }^{3}$

Il y aura donc à établir ce qu'il en est d'une société despotique "en général" afin d'en repérer la présence dans le judaïsme, afin aussi de comprendre comment le "Sujet » divin peut laisser la place, partiellement à un «Sujet royal». On assiste à cette captation relative du surnaturel en suivant l'évolution de la dénomination du roi. Ainsi le roi davidique est plus qu'un vassal ou un lieutenant de YHWH, ce qu'impliquait le titre de "messie" porté par Saül. L'oracle d'investiture royale transcrit à Psaume II,7 présente le roi comme un fils de YHWH : «Tu es mon Fils, c'est moi qui, t'ai engendré aujourd'hui ». Le Psaume LXXXIX, 28 ajoute que le roi est le fils aîné de Dieu. En effet tous les rois de la Terre dépendent de YHWH, mais c'est celui d'Israël qui est l'élu de Dieu (Psaume LXXXIX,4). Et de même que YHWH commande à l'univers entier, de même le roi, son représentant terrestre, règne, en droit, jusqu'aux extrémités de la Terre. Avec l'institution d'une monarchie héréditaire, Israël sera assimilé aux autres nation despotiques. Par ailleurs la signification de l'Alliance sera modifiée : l'éternité garantie au lignage de David, est une alliance (berit). L'Alliance devient un serment gratuit, un engagement unilatéral de Dieu. David (II Samuel XXIII,I-7) : «Dieu m'a accordé une alliance perpétuelle ». Psaume LXXXIX,4-5: «J'ai conclu une alliance avec mon élu. J'ai fait un serment à David mon serviteur : J'établis pour toujours ta postérité et de génération en génération. J'édifie ton trône ». Il conviendrait de déterminer si entre la non subjectivation essentielle du narrateur mythique et l'individuation $\mathrm{du}$ discours prophétique, il y a des étapes "intermédiaires" - une montée du "Je" en quelque sorte - ce qui ne postule en rien une continuité entre ces étapes, mais 
probablement plutôt des discontinuités. Même problème en ce qui concerne la distance entre le mythe qui pointe vers des Ancêtres en les mettant en scène et le style apodictique des interventions du "Sujet» divin YHWH énonçant la Loi. Comment par ailleurs articuler l'anonymat du Dieu avec son statut de suzerain d'Israël ? Rappelons que c'est un souci assez commun des sémites occidentaux de laisser le dieu dans l'anonymat. Ainsi une inscription araméenne $d u-V^{e ̀ ~ s i e ̀ c l e ~ d e ́ c o u v e r t e ~ a ̀ ~ T e i m a ~(A r a b i e ~}$ du Nord) rappelle l'existence à cet endroit d'un temple de Salm. Ce nom signifie selon toute vraisemblance "image". Une autre manière de sauvegarder l'anonymat du dieu consiste à l'appeler d'un titre précisant sa fonction. Ainsi chez les Arabes du centre: Yaghouth : «il porte secours »; Ya'ouq : «il protège ». D'une manière générale, le dieu dont le nom demeure caché, peut être le protecteur du groupe humain ou son guide, le défenseur d'un Etat, il peut être aussi le soutien et le secours d'un individu : plusieurs textes nabatéens et des inscriptions grecques provenant du même milieu sont des dédicaces du "dieu d'un tel». On peut rapprocher ce genre de désignations de la profession de foi biblique au "dieu des pères »4. De même chez les Arabes du Sud le nom des dieux se dissimule souvent derrière un titre, un nom abstrait: Wadd paraît signifier "amour" ou une désignation topique. De nombreux noms divins commencent par Dhou : "celui de", ou Dhat: "celle de". D'une manière générale, les noms propres ouest-sémitiques sont souvent de courtes phrases exprimant une relation entre la divinité et le porteur du nom, ou le bien qu'il lui a fait. Certains constituent de petites doxologies. Nous apprenons ainsi que le dieu, quel que soit son nom était le frère, le père, le seigneur, le sauveur de l'homme, qu'il était sa "lumière", qu'il lui accordait ses dons, sa grâce, sa bénédiction ${ }^{5}$. En ce qui concerne Israël, le nom de dieu noté par les quatre consonnes paraît remonter à un passé très lointain. Les Massorètes n'ont pas noté les voyelles du tétragramme, qu'ils lisaient adonay: "le seigneur". La critique moderne propose de le prononcer/yahwèh/et en fait une forme archaïque du verbe être "il est" (yihyéh). D'autres étymologies ont été proposées : "il fait être", "il souffle", "il abat", "il est passionné". Mais la prononciation /yahwèh/ du tétragramme n'est pas attestée avant le III siècle (Clément d'Alexandrie), temps où l'on avait oublié la lecture antique du nom divin et où l'on a pu chercher à le faire rimer avec le verbe être, en vertu d'une interprétation contestable d'Exode III,14, comprenant : "je suis celui qui est » au lieu de " je suis qui je suis », simple refus de répondre. L'anonymat est ainsi en quelque sorte redoublé. Le « Sujet absolu » est Zéro. Le «Sujet absolu» se manifeste tel quel. Ainsi le préambule du décalogue : "Je suis YHWH ton dieu, qui t'ai fait sortir du pays d'Egypte, de la maison des esclaves» (Exode XX,2). La fonction royale de YHWH est mise en lumière, il est présenté à l'instar d'un souverain qui, avant d'édicter sa volonté pour lui donner force de loi, se présente en quelque sorte lui-même. Remarquons que le célèbre code de Hammourati s'ouvre ainsi par une longue préface dans laquelle le roi babylonien donne sa titulature. Mais il s'agit d'une tradition sémitique d'attribuer au dieu une qualité royale et par conséquent la conduite de la collectivité humaine. Une telle conception s'exprime avec éloquence dans l'inscription de Mésha. source unique pour la connaissance de la langue et de la religion d'un peuple voisin des Hébreux, les Moabites, au -IXè siècle. Le roi Mésha rend grâce à son dieu Kamosh de la victoire qu'il a remportée sur les Hébreux. Si Omri, roi des Hébreux a paru d'abord l'emporter, c'est que Kamosh était irrité contre son propre pays. Mais Kamosh a rendu sa faveur à Mésha. Il l'a guidé dans sa campagne, qui est une véritable guerre sainte, et, chassé devant lui l'envahisseur. Ce nom divin désigne la divinité active par excellence. Le « Sujet absolu » est ainsi à la source du bien comme du mal et 
il existe une situation constante d'échange symbolique entre lui et la société. C'est l'analyse de cet échange ou de ce mode spécifique de déterminer l'autre de la société, qui devrait nous retenir. Il ne semble pas que le «Sujet absolu» découle de l'instauration d'une royauté terrestre, et ce nécessairement, dans la mesure où, par exemple, le surgissement de la royauté en Afrique n'a pas entraîné celui d'une divinité royale. Ce qui revient à dire que l'émergence d'un pouvoir terrestre effectif (coercitif) doit amener une transformation du champ symbolique, mais qu'il n'en découle pas nécessairement l'institution d'un « Sujet absolu ». D'autre part, le phénomène judaïque est bien singulier : il suffit de considérer que les fautes de David rejaillissent sur le peuple. Ainsi de l'histoire de l'holocauste effectué par David sur l'aire d'Arawnah le Jébuséen pour écarter la peste de Jérusalem (II Samuel LXXIV). Or la peste a été envoyée par YHWH afin de punir la faute de David qui a ordonné le recensement d'Israël. On sait que la tâche de tout nouvel Etat est de quantifier la population afin de pouvoir organiser l'armée et de pouvoir faire payer l'impôt. Ce que YHWH sanctionne donc c'est proprement le principe de la royauté. Or le châtiment retombe sur le peuple dont le roi fait œuvre de propitiation et d'expiation. La faute est probablement davantage celle d'Israël que de son roi : ce qui est sanctionné c'est la revendication d'une médiation terrestre telle que la séparation de la société d'avec sa Loi ne pourra être qu'opacifiée. Ainsi ce n'est peut-être pas par hasard que le Chroniste remplace YHWH par Satan pour expliquer l'origine de l'initiative de David (I Chroniques XXI,I). On peut donc, à partir du peu que l'on sait des sociétés ouest-sémitiques, établir ce en quoi Israël s'en distingue. La conception d'une royauté terrestre leur est probablement commune. Ainsi d'après une inscription minéenne, un roi s'accuse auprès du dieu Athtar parce que des documents ont été dérobés dans le temple, et parce qu'une ordonnance royale a été violée. Le roi ne peut être directement coupable de tels forfaits, mais il en est responsable et prend sur lui le poids d'une faute commise par ses sujets. Il agit ici en qualité de représentant de la collectivité auprès des dieux. C'est l'aspect le plus important de la fonction religieuse de la royauté en Arabie du Sud. Par ailleurs, le roi est le représentant des dieux auprès des hommes. Divers documents invoquent le roi aussitôt après les dieux énumérés en tête des textes; surtout le roi paraît être mis en relations personnelles avec la divinité nationale : le roi de Qataban était appelé "premier né de Amm" et celui des Minéens: "fils de Wadd". D'autres documents témoignent que Qatabanites et Minéens se considéraient comme des enfants de leurs dieux nationaux. Cela permet-il de rendre compte du fait que dans toutes les cultures sémitiques, il est difficile de séparer le droit et la morale de la religion et que toute faute est plus ou moins un péché ? On doit pouvoir affirmer l'existence d'une Décision instituante commune à toute cette aire culturelle, mais il n'en reste pas moins que la spécificité d'Israël est avérée.

\section{La religion, phénomène idéologique?}

4 Il nous semble qu'au préalable, il faudrait revenir rapidement sur la nature de l'idéologie afin de limiter l'extension de son concept, de telle manière qu'il ne puisse pas englober tous les systèmes de représentations sociales existants ou possibles et que l'on puisse spécifier l'essence du mode de penser religieux. Nous emprunterons à $\mathrm{Cl}$. Lefort une problématique de l'idéologie permettant, à rebours, de cerner un peu ce qu'il en est de la représentation dans des sociétés que nous nommerons, provisoirement, despotiques, "théologico-politiques». Il convient d'emblée de 
circonscrire l'idéologie à un type de société, donc de refuser son emploi en ce qui concerne les sociétés primitives, féodales et despotiques. Dans ces sociétés, le discours dominant tire sa légitimité d'une référence à un ordre transcendant, la "réalité" sociale ne se donne pas comme directement intelligible. Par ailleurs, au sein même de la société historique, il faut distinguer, et ce contre Marx lui-même, ce qui est strictement de l'ordre de l'idéologique, donc de l'imaginaire, de ces pôles constitutifs de la société, que sont ceux du pouvoir, de la loi et du savoir, cet ordre du symbolique sans lequel la réalité sociale ne serait pas intelligible pour les hommes. Ce langage dans lequel se conçoit et se réalise la pratique sociale n'est en effet pas réductible aux conséquences de la division du capital et du travail ou à un certain état de développement des rapports sociaux. Au contraire,

«ces rapports ne s'agencent, ces effets ne se développent qu'en fonction de conditions que nous ne saurions mettre au registre du réel, en revanche le "réel" s'ouvre aux hommes, s'ordonne, devient lisible une fois posés les repères d'une nouvelle expérience de la loi, du pouvoir et du savoir, une fois inauguré un mode de discours dans lequel certaines oppositions, certaines pratiques de fait s'avèrent, se renvoient les unes aux autres, contiennent virtuellement un sens universel, en permettant un échange réglé entre l'agir et le penser. $»^{6}$

L'apparition de l'idéologie suppose une désintrication de l'ordre social et de l'ordre du monde, et ce qui va de pair, une désintrication du politique et du mythico-religieux. Cette rupture, coïncidant avec l'émergence de l'Etat moderne, a été pensée par les humanistes de la Renaissance, sans que par ailleurs l'Humanisme puisse être réduit à de l'idéologie ${ }^{7}$. Alors, le discours fait en lui-même l'expérience de l'altérité, au lieu que la parole s'ordonne sous le pôle extérieur de l'autre. La société ne trouvant plus dans l'audelà son sens, est conduite fondamentalement à reconnaître la division sociale qui la déchire, et ce qui lui est liée, la division temporelle : sa propre historicité. Mais cette reconnaissance ne peut s'achever, l'étrangeté doit être maîtrisée : l'idéologie est ce discours (cette pluralité de discours en fait) qui annule les effets de la division et restaure le "réel". Ce faisant elle ne peut que méconnaître les conditions de son propre engendrement, c'est-à-dire la division entre la pratique et le savoir qui en rend compte. Pour cerner ce qu'il en est du symbolique, on peut suivre ce que dit Marx des rapports existants, dans la société indienne, entre les communautés paysannes et le Prince ou le Despote ${ }^{8}$. Une société ne peut exister comme société qu'à la condition de se forger la représentation de son unité. C'est dire qu'une société ne saurait être simplement parce que ses membres, malgré la séparation de leurs activités, échangent. Pour Marx, des "rapports sociaux bornés", sont à l'origine du détachement du réel d'une communauté imaginaire, laquelle parce qu'elle échappe à la prise des hommes va exercer un véritable pouvoir sur eux, en se figeant, en convertissant les distinctions sociales en différences naturelles, le particulier étant travesti sous les traits de l'universel, et l'historique éliminé par les prestiges de l'essentiel. L'ordre social "terrestre" se trouve ainsi légitimé ; dominés et dominants se voient assurés dans leurs statuts, les individus trouvant les normes de leur existence dans la représentation de la communauté imaginaire. Mais on ne saurait ramener la dépendance de la vie ici-bas à un ailleurs, à une seule légitimation de la domination. Marx affirme que le Mode de Production Asiatique - caractérisant plus particulièrement les Indes et la Chine - tend à se reproduire tel quel, échappant à l'événement. L'organisation sociale est pétrifiée du fait de la séparation radicale des communautés paysannes et de la communauté imaginaire. C'est reconnaître l'efficacité symbolique du despotisme, et d'une manière générale le pouvoir de la représentation. Mais cet assujettissement des hommes à la parole 
religieuse ou mythique, tel que la division sociale ne peut se développer et travailler la société qui se caractérise par son conservatisme, tel aussi que l'invention technique ne puisse remettre en cause l'ordre social, n'a pas sa raison seulement du côté de la défense des "intérêts" de la caste ou du groupe dominants. Si le nouveau ne peut pas faire irruption, à moins d'avoir été conçu au préalable, c'est que la séparation entre l'ordre de la loi, le discours sur le social et le pouvoir et l'ordre de la "réalité", est radicale. Tout se passe comme si constamment la communauté imaginaire modelait la "réalité sociale", de manière telle que les différences sociales apparaissent comme naturelles et définitives. On voit donc ce qui distingue ce dispositif symbolique de l'idéologie, puisque dans le cas des sociétés despotiques la dissimulation de la division sociale coïncide avec le pouvoir effectif d'enrayer ses effets, et la dissimulation de l'historique avec le pouvoir effectif de barrer la voie du changement. Ainsi donc. le processus idéologique est différent du processus religieux: parce qu'il tend à se développer dans les limites de l'espace social, ce faisant il a partie liée avec la connaissance scientifique prétendant à l'autodéchiffrement du réel; parce qu'il est soumis aux effets du bouleversement social incessant qu'engendre le capitalisme, il doit accomplir son œuvre d'occultation de la division en modifiant ses propres énoncés ou en ayant recours simultanément à une multiplicité de représentations pour colmater les brèches que le changement ouvre dans la "rationalité du réel »'. Marx renvoie l'idéologie du côté de l'imaginaire, du côté de la dissimulation. Mais si elle est régie par un principe d'occultation, il n'en reste pas moins que celui-ci fonctionne aussi dans les sociétés pré-capitalistes, lesquelles se référant à un ailleurs ne peuvent que se manquer dans le savoir d'elles-mêmes.

6 L'idéologie entretient donc une relation singulière avec la société historique. En outre, elle suppose un régime de l'imaginaire tel que celui-ci ne s'inscrive plus dans le dispositif symbolique de la séparation du discours sur le social d'avec la société, impliquant que les hommes trouvent sens à leur existence dans un ailleurs. Il y a idéologie quand surgit la question de l'engendrement du social depuis son lieu propre. Et cet engendrement doit être contenu par un discours qui en neutralise les effets. L'idéologie a donc un rôle de conservation, rétablissant en quelque sorte la place de la société non-historique dans la société historique elle-même. Selon Lefort qui distingue idéologie "bourgeoise" dont l'apogée aurait eu lieu au XIX mais aussi de l'idéologie moderne, cette première forme historique pourrait être caractérisée par quelques traits. Ce discours vit de la négation de toute référence à la transcendance, mais s'ordonne en fonction d'un clivage entre les idées et le supposé réel. Une certaine transcendance est ainsi restaurée et, note-t-il, c'est un discours qui s'écrit avec des majuscules. Les "Idées" (la Nature, l'Humanité, le Progrès, l'Art, la Science...) sont à la fois des représentations et des normes. La règle est ainsi constamment exhibée, proclamée comme régissant les choses mais aussi comme ce qui doit être suivi et repris dans la pensée et l'action. Avec la règle est produite la figure d'un garant, et c'est toute une imagerie qui se développe alors. Il y a un maître, qui dans chaque cas, déclare ce qu'il faut faire et ce qu'il faut penser. Le discours pédagogique sera le plus représentatif de ce régime de l'idéologie, fixant dans un champ délimité - l'éducation - une figure correspondante de l'autre qui hante toujours ce type de discours : l'enfant ici. Ce discours produisant des Idées qui à la fois sont des normes, relevant d'une certaine transcendance, et en même temps des représentations du réel, connaît une contradiction constante. En effet, il ne se borne pas à décrire ce qui est, l'état de telle institution qui pour exister se représente, il tente de recouvrir ce 
discours immédiatement produit par les agents, par une "vue" de l'institution, par ce qu'elle devrait être si elle devait coïncider avec sa nature. Ainsi, dans tous les lieux de l'espace social, s'affirmeront des convictions produisant un imaginaire du rapport social. Mais ces convictions sont toujours menacées par la dérive constante des institutions qu'elles sont à la fois censées représenter et normer. Elles doivent se modifier sans cesse jusqu'à ne plus pouvoir affirmer que le caractère sacré de telle institution (exemple du discours idéologique sur la famille). L'idéologie trouve alors sa clôture dans la tautologie.

\section{Problématique du pouvoir chez P. Clastres}

$7 \quad$ La philosophie du pouvoir de P. Clastres se développe dans plusieurs textes réunis dans La société contre l'Etat, mais seule une lecture de la Chronique des Indiens Guayaki permet de lui accorder sa réelle portée. Par ailleurs, la préface au livre de M. Salhins Age de pierre, âge d'abondance semble ouvrir les nouvelles perspectives. Selon l'auteur, le texte intitulé : Echange et pouvoir : philosophie de la chefferie indienne, publié en 1962, étant trop sous l'influence de la théorie structuraliste de l'échange de Lévi-Strauss, ne devrait être cité que pour mémoire. De fait la préface au livre de Salhins reviendra sur la question des rapports d'échange entre la société et le chef ; mais il nous semble que les conclusions de cet article -"de jeunesse" - doivent être rappelées. Quelle est la "fonction" de ce chef, de ce pouvoir politique qui n'est pas coercitif, qui ne saurait entraîner son peuple à sa suite dans une guerre contre une autre tribu si celui-ci n'en a pas décidé ainsi, qui ne saurait imposer ses propres décisions concernant la vie de la tribu? Ce pouvoir n'est rien, n'est que symbolique. Mais il a en lui-même la possibilité toujours réelle de devenir réel. A la suite de l'analyse des rapports d'échange fondant l'extériorité du pouvoir, ou sa transcendance limitée, par rapport à la société, Clastres montre que du fait de son statut, le pouvoir est rejeté du côté de la nature. Il est la négation de la société, de même que la nature est la négation de la culture. Mais cette négativité du pouvoir est elle-même niée, en ce qu'elle reste contenue. Etant hors société, le pouvoir ne peut que se retourner contre la société. Les sauvages n'ont pas eu besoin de faire l'expérience de cette prise de pouvoir, il serait vain de se retourner vers un quelconque événement de cet ordre pour rendre compte de l'impuissance structurelle de la chefferie indienne, même s'il est avéré que des chefs aient joué au chef, tentant de mener par exemple des guerres de vengeance personnelle. Ce qui est en jeu, beaucoup plus que les conséquences d'une expérience dramatique - la transformation d'une chefferie en pur despotisme - ou les résultats d'une intuition "sociologique" concernant l'essence du pouvoir, est l'effet d'une décision affectant le rapport du pouvoir à la société, du savoir à la société et du savoir au pouvoir lui-même. $C^{\prime}$ est dire qu'on ne peut rendre compte de l'impuissance de la chefferie en n'analysant seulement que des mécanismes d'échange, et ceci, même en termes de flux et de rabattement sur les flux comme chez les auteurs de L'Anti-Oedipe.

8 Notons, à la suite de Clastres, qu'en dehors d'une "fonction" symbolique, essentielle, qui reste, à ce niveau, énigmatique, ce que sont les effets, dans le réel, dudit pouvoir. Les tribus indiennes étant constamment en guerre, le rôle du chef est aussi de conduire les hostilités, ce qui suppose une compétence certaine. Rappelons que cette fonction peut être reprise par un chef de guerre qui ne se verra obéi que le temps des combats. A l'opposé de Lévi-Strauss ne voyant dans la guerre sauvage qu'une rupture momentanée de l'échange, Clastres y décèle l'exercice de la division, producteur du multiple, sans 
lequel les sociétés sauvages ne sauraient échapper au règne de l'Un. En effet, la société contre l'Etat, est aussi une société de la fragmentation, une société constamment travaillée par les mouvements centrifuges, où donc, en cas de conflits internes à la société, il est toujours possible pour tel groupe de faire sécession et de faire acte de fondation ailleurs. Chef de guerre face aux ennemis, le chef est aussi celui qui représente son peuple, celui qui conclut des alliances, un ambassadeur en quelque sorte, ce qui suppose aussi une habileté certaine. Ces fonctions, mais aussi la nonréciprocité existant dans les échanges entre lui et la société, peuvent permettre de rendre compte de sa polygamie, polygamie marquant sa différence avec la société, même si celle-ci la pratique aussi. Dans ce sens, puisqu'il est l'autre de la société, il est aussi le support de l'imaginaire social, ou son point d'ancrage, étant ainsi celui qui échappe aux règles constitutives de la société, celles de l'échange des femmes. Ce qui peut exprimer,

" au-delà même de l'attachement de la culture à ses valeurs, l'espoir ou la nostalgie d'un temps mythique où chacun accéderait à la plénitude d'une jouissance non limitée par l'exigence de l'échange; l'opulence du chef est le songe éveillé du groupe. $»^{10}$

9 A l'intérieur de la société, le chef est celui qui dit la loi. Puissance conservatrice de la chefferie qui caractérise la société primitive. Le chef réaffirme la nécessité de conserver l'ordre social, de faire comme les ancêtres l'ont toujours fait. Or, ces ancêtres qui sont à l'origine de la loi, de cet ensemble de règles déterminant l'ensemble des activités de la société et des individus, ne sont pas les ancêtres réels, mais les fondateurs mythiques de la société, les législateurs. Ce conservatisme concerne l'être social tel qu'il a été institué par les ancêtres.

10 Ainsi le chef ne dit-il rien de plus que ce que tout le monde sait déjà. Discours sans contenu. Parole qui ne saurait être de commandement, d'ailleurs les autres ne l'écoutent même pas : chacun vaque à ses occupations. La parole du chef n'est pas dite pour être écoutée. Mais il ne pourrait échapper à ce devoir-là. Le silence le condamnerait, il doit montrer son pouvoir sur les mots. Et Clastres ajoute :

«la société primitive est le lieu du refus d'un pouvoir séparé, parce qu'elle-même, et non le chef est le lieu réel du pouvoir. La société primitive sait par nature que la violence est l'essence du pouvoir. En ce savoir s'enracine le souci de maintenir constamment à l'écart l'un de l'autre, le pouvoir et l'institution, le commandement et le chef. Et c'est le champ même de la parole qui assure la démarcation et trace la ligne de partage. En contraignant le chef à se mouvoir seulement dans l'élément de la parole, dans l'extrême opposé de la violence, la tribu s'assure que toutes choses restent à leur place, que l'axe du pouvoir se rabat sur le corps exclusif de la société et que nul déplacement des forces ne viendra bouleverser l'ordre social. ${ }^{11}$

11 Mais qu'est-ce qu'une société qui est elle-même le lieu réel du pouvoir ? Est-ce au sens de la démocratie grecque, où l'espace politique est centré12 selon Vernant ou Detienne ${ }^{13}$ décrivant les assemblées de guerriers, s'installant en cercle, de telle manière que le centre, c'est-à-dire le lieu du pouvoir, reste vide, n'étant occupé momentanément que par celui qui va parler au nom du groupe de ce qui le concerne en tant que collectivité ? Alors effectivement il y a acte législatif et ce pouvoir de la société est su. Mais en va-t-il de même dans la société sauvage dont la loi est donnée par les ancêtres? Dans ce sens, si la société est le lieu réel du pouvoir, alors elle l'ignore. La société se pense comme recevant d'ailleurs son ordre. La coupure est totale entre cet ailleurs (les ancêtres, les héros culturels, les "dieux") et elle-même. La société pose à l'extérieur d'elle-même l'origine de son ordre social. Mais il n'y a pas en elle-même d'individus ou de groupes 
qui par leur fonction ou leur origine seraient dans une plus grande proximité de ce lieu de l'origine. Tous les individus sont également éloignés du lieu de l'origine et sont également dépositaires du savoir de l'ordre social, et en sont donc aussi les gardiens.

C'est dans ce sens que la société est le lieu réel du pouvoir.

L'énigme que rencontre P. Clastres est celle d'un type d'organisation sociale - la société sauvage - dont on ne peut ignorer qu'elle connaît une existence politique, et Gauchet parle justement de la " politique des sauvages ${ }^{14}$, mais qui en même temps se construit dans le refus du pouvoir. A ne s'en tenir qu'à La Société contre l'Etat, on acquiert la représentation d'un pouvoir politique éminemment cancérigène, et l'on comprend bien alors que la société fasse tout pour le neutraliser, pour le repousser dans l'ailleurs, livrant même des femmes afin de le mieux tenir. Mais l'analyse qui est faite des "fonctions" du pouvoir ne permet pas d'en épuiser la nécessité, la société pouvant fort bien s'accorder des représentants pour guerroyer ou pour négocier sans que ceux-ci deviennent de facto des figures de la chefferie. Par ailleurs si le chef est celui qui dit la loi, mais si sa connaissance ne peut par essence excéder la connaissance qu'en ont les autres membres, sa parole n'apportant aucune information nouvelle en cet ordre, il paye ainsi sa dette à la communauté en lui faisant don de mots, alors la négativité qu'il est pour la société tend à s'évanouir. A la limite, cette négativité - en creux - n'est concevable qu'en fonction de ce qu'elle pourrait être : un pouvoir politique coercitif, un Etat. Toutefois, l'extériorité même passive de la chefferie indienne, parce que cette dernière pointe vers la loi, est une constante réaffirmation de l'altérité radicale de l'origine de la société. Mais en quoi celui qui occupe ce rôle est-il gros d'une sécession, de telle manière qu'il puisse se retourner contre la société et prendre pouvoir sur elle? Notons qu'alors il ne serait plus celui qui rappelle l'origine, mais origine lui-même, ou tout au moins sans qu'il ait à se déclarer à l'origine de l'ordre social - configuration qui semble apparaître dans l'aire indo-européenne selon Meillet que cite Benveniste ${ }^{15}$, avec un nom de roi appartenant à un dialecte local du domaine linguistique iranien, qui signifie "celui qui est puissant par lui-même, qui ne tient que de lui-même son pouvoir" - dans une relation de descendance, de représentation singulière avec les ancêtres. Mais alors c'est la Décision (sociale) antérieure qui serait radicalement modifiée. En effet que le chef sauvage fasse le chef - qu'il prenne effectivement le pouvoir - ce ne sera pas seulement son rapport à la société qui sera modifié mais conjointement son rapport au savoir et le rapport de la société au savoir.

Un point semble malgré tout important dans cette prise de pouvoir, et que relève P. Clastres dans sa préface au livre salutaire de M. Salhins, c'est la question de la dette, dette qui pour lui est "signe et vérité du pouvoir" (p. 26). A sa suite, il reprend l'analyse du rôle du leader dans les sociétés mélanésiennes, dans la mesure où les ethnologues ont toujours vu dans le "Big-man" un vrai chef. Or, ce dernier, aidé pour ce faire par sa parenté qui se transforme ainsi en clientèle, travaille durement afin de produire des surplus de consommation qui seront distribués à la communauté. Nous savons que le chef pour être chef doit être un bon orateur et généreux. Mais on assiste à une "autoexploitation du big-man" alors qu'évidemment il n'a pas les moyens de contraindre la communauté à travailler pour lui. Ainsi, « la société en son ensemble exploite le travail de la minorité qui entoure le big-man »(p. 23). Il devient alors bien difficile de parler de pouvoir, ce qui conduit à ne voir dans l'activité du big-man que le moyen de se faire reconnaître dans sa prodigalité : il ne cherche que le prestige et non pas la satisfaction d'un désir de pouvoir. Dès lors faut-il, dans le cas de ces sociétés, disjoindre pouvoir et 
prestige. A ce niveau, l'échange est égalitaire: des biens contre une reconnaissance, mais ne suffit probablement pas à l'érection d'un big-man, car ce type d'échange pourrait concerner tous les membres de la communauté à l'intérieur du cycle général de l'échange-don, dont on sait qu'il peut être interprété comme mouvement de la reconnaissance ${ }^{16}$. Cette relation, en apparence égalitaire ne suffit pas en effet, elle dissimule une inégalité entre le leader et la société. Celui-ci a une dette envers la société en tant précisément qu'il est le leader. Et si les flux de biens vont vers la société, c'est qu'elle exerce le pouvoir sur le leader, dont elle se garantit ainsi de le voir rester à l'extérieur du pouvoir : il n'en deviendra pas l'organe séparé.

«Prisonnier de son désir de prestige, le chef sauvage accepte de se soumettre au pouvoir de la société en réglant la dette qu'institue tout exercice du pouvoir » (p. 25).

L'analyse du sens de la dette permettra ainsi de déceler qui de la société ou du "leader" exerce le pouvoir. Dans les royautés et ailleurs, les endettés sont ceux que les rois, grands-prêtres et despotes appellent "gens du commun". Le sens de la dette permet d'étalonner les sociétés selon la discontinuité : société contre l'Etat/société pour l'Etat. La pertinence de ce critère peut amenuiser notre compréhension du rôle et de la "fonction" du leader. D'autant que Clastres semble hésiter entre deux problématiques, cette ambiguïté ayant d'ailleurs rendu possible deux interprétations de son œuvre, l'une en termes de flux, de rabattement des flux, de codage. de disjonction et de conjonction, interprétation horizontale(superficielle dans ce sens) développée dans l' Anti-Oedipe. L'autre se référant davantage à une problématique issue de la psychanalyse, celle de Lefort. Difficile, notre tâche l'est assurément, de faire coexister un chef qui dit la loi et un chef qui menace la société de "flux de pouvoir" (p. 26). Or, si le Big-man est piégé dans son désir de prestige et qu'en lui se conjugue le désir de produire pour donner (prodigalité, satisfaction du narcissisme) et le devoir de produire pour payer sa dette, c'est qu'en même temps il est cette instance sans laquelle tous ces segments constituant le "mode de production domestique" ne pourraient tenir ensemble. Ce qui revient à dire qu'une référence commune à un ordre mythique ne saurait suffire. Le "chef" doit ainsi limiter l'extension des forces centrifuges dans la société, il représente la généralité contre la segmentarisation propre au M.P.D. :

«Le don ne saurait organiser la société sous forme solidaire, mais seulement sous forme segmentaire. La réciprocité est une relation "entre" deux termes. Elle ne dissout pas les parties séparées au sein d'une unité supérieure, mais au contraire conjugue leur opposition et par là-même la perpétue. Aussi bien le don n'institue-til pas non plus une tierce partie dont les intérêts prévaudraient sur les intérêts séparés des contractants. $»^{17}$

16 Face à une horizontalité radicale de la substance sociale, l'instance de la chefferie instaure un tenir-ensemble toujours menacé. Même ambiguïté en ce qui concerne la reproduction de la société sauvage. En effet le M.P.D. excluant la production de surplus implique que la société ne puisse se reproduire qu'identique à elle-même, elle ne saurait se lancer dans l'inconnu, la société sauvage contrôlant tous ses flux. Or, l'immanence à la société de son sens, celui-ci étant en même temps posé comme ayant son origine dans l'ailleurs, conduit au même résultat. Donc, est-ce le mode de production qui entraîne une non-historicité de la société - son caractère répétitif - ou est-ce son attachement à l'ordre mythique ? Société ne connaissant pas, car la refusant, la division entre l'Etat et elle-même, et en conséquence refusant en elle-même le partage entre les dominants et les dominés, la société sauvage pourrait apparaître comme une totalité pleine et indivise. Or, elle est traversée par une division interne 
puisque la dimension de l'échange conflictuel est fondamentale, mène si par ailleurs les acteurs du "conflit" ne sauraient remettre en cause les principes de l'échange luimême, puisque la société dénie à tout acteur humain la possibilité d'intervenir en tant que novateur dans l'ordre social et donc dans l'ordre du monde naturel ou surnaturel. Ainsi donc la société ne se sachant pas l'origine d'elle-même, mais projetant celle-ci dans l'ailleurs, dans un temps autre que le temps profane, dans un temps sacré, étant le fait d'ancêtres n'ayant rien à voir avec l'espèce humaine présente, cette société donc connaît une division fondamentale. Mais ce savoir d'elle-même, tout en étant fondé dans l'ailleurs, même si elle ne peut pas le reprendre dans un mouvement de réflexion, dans l'acte théorique, il n'en reste pas moins qu'il est présent dans l'immédiateté et constamment mis en œuvre. Ce savoir des choses de la nature, du monde des dieux, de la nature du lien social est su à fleur de peau. Son immédiateté est telle, que pour telle société sauvage, telle communauté, il ne peut y avoir d'autre société. Le singulier est l'universel. Les sociétés sauvages sont profondément ethnocentriques. D'où

«la propension qu'ont les groupes ethniques de s'auto-déterminer, s'autodénommer par un terme qui, dans leur langue, signifie ni plus ni moins qu'homme, gent ou quelque chose d'approchant. C'est très exactement le sens du mot "yanomami". L'ethnie c'est le point central de l'univers humain, c'est l'humanité par excellence, autour de laquelle tout doit nécessairement converger ou graviter. Pour un Yanomami, tout ce qui n'appartient pas à son propre monde socioculturel est forcément étranger, nabê. Les mots yanomami et nabê forment couple et s'opposent à la fois [...] ce sont les ennemis, car de fait, l'étranger est bien un ennemi potentiel [...] ravalé au rang d'une sous-humanité méprisée et crainte tout à la fois. $»^{18}$

La société connaît donc une division radicale externe, dont la conséquence est qu'elle ne peut être que "pour la guerre". On comprend mieux aussi que ces sociétés "sans écriture", donc sans un corps de scribes, soit aussi par excellence des sociétés de l'écriture. En effet la loi est écrite, mais sur les corps. Cette écriture, Clastres l'identifie dans les marques imposées au cours des séances d'initiation des adolescents. Il s'agit essentiellement de laisser des traces, de marquer les corps et ce pas seulement pour éprouver le courage des futurs guerriers ou pour leur rappeler leur appartenance à la société, mais afin d'affirmer l'immédiateté de la loi. La loi est d'autant mieux sue qu'elle est plus proche. Le corps ne pourra jamais en perdre le souvenir. Sa distance à la singularité étant nulle, elle ne peut être prononcée, elle n'a pas besoin d'être dite. Dès lors personne parmi les hommes ne pourra se dire du côté de la loi, dire la loi et la faire respecter. Elle est loi égale pour tous. Loi absolument non séparée puisque nul n'a prise sur elle. Personne ne la représente ou n'en est le gardien. Cette loi est éprouvée, nul besoin de l'exprimer. Cette écriture sur les corps est elle-même contre l'écriture séparée, celle des scribes et de leur Etat. Clastres ${ }^{19}$ ouvre ainsi la voie à une politique de l'écriture, dégagée du fonctionnalisme. Remarquons que le rapport de la société au savoir détermine le mode d'inculcation de ce savoir, et donc aussi les facultés humaines en jeu. La pédagogie initiatique d'une société sauvage a pour corrélat la torture du corps des adolescents, il s'agit de cultiver la mémoire la plus profonde. L'oubli de la loi ne sera pas possible. A l'inverse, puisque ce sont des sociétés qui tout en étant traversées par l'histoire, sont en quelque sorte non-historiques, l'événement doit être maitrisé afin de ne pas remettre en cause l'ordre social. C'est dire que la culture de la créativité pour la créativité n'a pas de sens. Au contraire, un type de société se pensant comme historique, accueillant l'événement, le désirant, sachant que la société est à l'origine d'elle-même et que rien d'autre qu'elle-même ne saurait la fonder, mais qui 
corrélativement ne peut se saisir dans l'immédiateté, étant étrangère à son propre ordre se faisant dans l'obscurité, ce type de société donc ne peut se comprendre luimême que dans l'élaboration de la théorie, et n'ayant pas à s'identifier à un ordre donné d'avance et ailleurs, ne peut s'abandonner qu'au mouvement infini de la recherche. Ce qui sera alors cultivé, tant dans des établissements spécialises (écoles) qu'en général, ce seront toutes les facultés liées à la créativité et à l'innovation. Si l'on veut bien considérer que l'éducation est à la fois initiation et instruction (acquisition de connaissances), on admettra que l'on peut classer les sociétés selon l'importance accordée à l'initiation et inversement à l'acquisition de connaissances, étant entendu que les deux facteurs vont toujours ensemble, qu'il n'y a pas d'initiation dénuée de sens et à l'opposé d'inculcation d'informations sans modification de l'individu. Les sociétés despotiques doivent constituer un groupe intermédiaire, étant des sociétés de la loi écrite.

18 L'immédiateté de la loi ou sa réalisation immédiate par l'ensemble de la société, son accrochage dans l'avant et l'ailleurs, sont ainsi des éléments de l'appareil grâce auxquels la chefferie ne peut pas devenir effective, l'Etat de se constituer et se retourner contre la société. Mais le prix à payer par les "sauvages", c'est de se savoir collectivement ou individuellement impuissants en ce qui concerne leur ordre social. A l'inverse, les sociétés étatiques modernes se savent à l'origine d'elle-même, elles se sont émancipées de la sphère du religieux. Rien dans la société qui ait raison ou cause autre que sociale. Dès lors, l'Etat a le champ libre et peut réaliser ce projet présent dès ses origines: celui d'une prise en charge totale de la société jusqu'à l'utopie d'une réconciliation dans l'indistinct avec la société. Mais de l'Etat despotique à l'Etat totalitaire un pas immense est franchi. C'est que le despote n'a pas en lui-même le principe de sa légitimité. Cette légitimité, il la tire de ce qu'il représente ou est l'héritier d'un ordre qui lui demeure transcendant. Il peut très bien être ce sans quoi l'ordre social ne pourrait fonctionner, il ne peut se poser comme l'origine effective et omnisciente de l'organisation sociale. Il trouve toujours sa limite dans sa référence nécessaire au religieux. Ce n'est qu'avec la disparition de tout garant religieux que l'Etat peut donner toute sa mesure :

"Caution efficace de l'ordre et du pouvoir établi, la justification de l'ici-bas par un au-delà n'en continue pas moins à jouer d'autre part un rôle aussi occulte que considérable de neutralisation du pouvoir. $»^{20}$

Il serait donc important de comprendre les mutations dans l'ordre religieux qu'impose l'émergence de l'Etat. Une telle possibilité est en effet ouverte dès que parmi les hommes, l'un d'entre eux peut se dire le représentant de l'ailleurs. N'est-ce point le cas du chamane qui participe nécessairement aux "forces" de l'ailleurs ou le cas du devin dont les pratiques permettent de faire le point en ce qui concerne les divinités qui peuvent influer sur la vie de l'individu? Or, l'un et l'autre, ne sont pas des représentants du monde autre, quelle que soit leur possibilité d'interprétation et d'action; ils sont dans un même éloignement de l'ailleurs que l'ensemble de la société. Ce n'est que lorsque le surnaturel descend sur terre par l'intermédiaire d'un prophète par exemple, que se constitue à l'intérieur de la société un foyer capable de la bouleverser. Il semble que l'on puisse circonscrire cette rupture en suivant l'analyse $\mathrm{d}$ 'H. Clastres des prophètes Tupi-Guarani ${ }^{21}$. Paradoxalement ceux-ci n'apparurent qu'en réaction à une véritable prise de pouvoir des chefs Guarani, leur message prophétique devant amener les hommes à quitter cette terre habitée dorénavant par le mal. Or ce prophétisme est proprement révolutionnaire : il faut, selon lui, cesser de travailler, 
cesser d'échanger les femmes et partir pour atteindre un lieu terrestre : la Terre sans mal. Or les prophètes sont dans une relation individuelle avec Dieu, - fils de Dieu -, ils multiplient dans leur comportement les signes de la différence, se tiennent à l'écart, se font précéder d'une troupe : autant de moyens destinés à renforcer leur appartenance au surnaturel. Il est vrai que l'essentiel de la révolution qu'ils professent est mystique, qu'il ne s'agit pas tant de bâtir un autre monde que de se transformer soi-même afin de devenir "léger". Il n'en reste pas moins qu'avec eux surgit la figure d'un pouvoir capable d'interpréter les volontés divines, d'en être le seul initiateur et donc l'unique référence ici-bas de l'au-delà. De la société va se détacher un organe, lequel prétextant sa filiation privilégiée avec l'ailleurs va se donner comme origine du sens, sachant ce qui est bon et juste pour le monde des hommes et héritant de l'altérité propre au surnaturel, se faisant adorer. Il sera donc important d'établir la succession des figures du « Sujet » social, du roi divin au Dieu « Sujet absolu » d'un peuple ou de l'univers. A ce point, il convient de rappeler que le discours social, dans la société primitive est anonyme. Celui qui dit le mythe ou explique le sens d'un rituel n'exprime qu'une parole collective, même si reprenant le mythe, il le modifie. C'est cet essentiel anonymat qui disparaîtra avec l'apparition du prophète dont la particularité s'oppose à l'anonymat de la parole collective. La société sauvage n'est pas un "sujet", au sens où, tout en pouvant donner une signification à son activité constante d'institution du social, elle ne peut pas réfléchir celle-ci. Il faut mettre en rapport cette irréflexion avec l'altérité de son origine. Puisque la société reçoit d'ailleurs le sens de son être, elle se coupe de la possibilité de réfléchir son activité instituante. Puisque dans la société, chacun est capable, au moment où il le faut, de savoir ce qu'il doit faire, à égalité avec les autres et que ce devoir lui vient des ancêtres qui ont toujours agi ainsi, il n'est donné à aucun d'entre eux de reprendre à son compte le sens de cette activité en la réfléchissant. L'irréflexion foncière de l'activité instituante qui est la conséquence de la scission radicale de la société d'avec son origine, permet de comprendre, suivant P. Clastres, que le pouvoir est rabattu sur la société. Mais il faut ajouter immédiatement que ce pouvoir ne se sachant pas tel, la société sauvage ne peut pas devenir "Sujet ", de même qu'elle exclut l'apparition d'un quelconque sujet particulier, d'un intermédiaire entre son sens et son origine, à moins, comme nous l'avons vu, que la société soit ébranlée dans son organisation.

«Tout coup porté à l'anonymat du procès social est voie frayée à l'avènement du sujet social scellant cette fois catégoriquement la rupture avec l'ordre primitif et consacrant l'existence d'une division sociale encore incertaine peut-être en la personne d'un Roi/magique/doué surtout de puissance symbolique, mais affirmé avec le retranchement d'un despote appuyant sa toute puissance sur la violence. $»^{22}$

C'est dire qu'il conviendrait d'envisager un autre type de société, que l'on pourrait situer entre l'organisation primitive et la nôtre. Société qui ne serait plus contre l'Etat, mais qui malgré tout, continuerait de se penser encore en partie en fonction de l'ailleurs. Société que l'on peut nommer despotique, puisque aussi bien société de la division interne, celle existant entre le despote et ses sujets, celle séparant les sujets, faisant se dresser une partie de la société contre l'autre, ce qui suppose un renversement du sens de la dette. Or, la société despotique se référant pour l'essentiel à un ailleurs transcendant ne peut se réfléchir : la division interne qui la déchire ne peut lui apparaître, elle n'est pas pensable. De même que dans l'organisation primitive, l'ordre des représentations (mythes, récits, etc.) ne se borne pas à rendre compte de la société, il l'informe proprement, le sens de la société lui est aussi immanent. Mais alors, 
à la différence de l'organisation primitive qui elle, ne connaît pas de division interne, cet ordre social transcendant qui s'applique dans l'ici-bas produit des divisions sociales (ordres, castes). On sait la difficulté rencontrée dans l'interprétation des sociétés despotiques : faut-il les décrire suivant ce qu'elles disent d'elles-mêmes ou faut-il y voir des sociétés de la lutte de classes? Or, il est fort possible qu'elles soient à la fois des sociétés divisées - il y a bien un conflit entre le despote et la société, un conflit entre une partie de la société et l'autre - et d'autre part des sociétés d'ordres et de castes, ceux-ci ayant un fonctionnement effectif. Comme si donc la division interne ne pouvant être réfléchie, à l'exemple de la société moderne, et donc d'une certaine manière reconnue, devait être plaqué sur la réalité sociale un ordre des différences, ordre enraciné lui-même dans l'ailleurs. Cet ordre aurait donc une fonction de masque et ce masque agirait en informant la réalité sociale. Il convient de ne pas utiliser ici la notion d'idéologie, qui ne concerne que la société moderne, dans la mesure où l'idéologie est bien ce qui recouvre le conflit, mais qu'il n'en reste pas moins qu'elle le manifeste ${ }^{23}$. Par ailleurs le discours idéologique est un discours sur I'objet, celui qui l'emploie sait qu'il utilise un discours, et que celui-ci est à distance de ce qu'il vise. Au contraire le discours de la société despotique s'applique directement à l'objet, son adéquation au "réel" est totale : c'est le discours du réel, et non sur le réel. C'est un discours qui ne renvoie pas de l'intérieur à son statut de discours, et donc à sa différence avec ce sur quoi il est discours, et donc à une distance du locuteur discourant à son propre discours. Ce qui a pour conséquence qu'il échappe à la possibilité de la critique. Il conviendra probablement de nuancer ceci quand nous aborderons le cas d'une religion révélée : le Judaïsme. La catégorie du "Sujet » omniscient et omniprésent sera essentielle, ce qui ira de pair avec l'apparition d'une classe de prêtres et de savants prétendant s'arroger une partie de cette omniscience et de cette omnipotence. Nous avons vu que la pensée sauvage ne pouvait se constituer comme pensée-sujet. Il conviendrait, à la suite de Lévi-Strauss, d'en reprendre l'analyse afin de préciser ce qu'il en est de sa nature et de son statut. Ce qui frappe d'emblée c'est qu'il s'agit d'une pensée totalisante. Elle met systématiquement le monde en ordre, et fait correspondre les différents ordres entre eux. Mais ce qui peut sembler paradoxal dans ce souci d'exhaustivité, c'est qu'il ne fait pas retour sur lui-même dans un mouvement réflexif : c'est une totalisation sans totalité. La catégorie de totalité n'apparaît pas. Ainsi, suivant Clastres, il ne semble pas que les Tupi-Guarani connaissent la notion du Tout ${ }^{24}$. Pensée donc qui se développe sans s'achever, sans se donner un terme, et ce non par une quelconque faiblesse, mais parce qu'elle exclut la possibilité d'un Tout-Sujet. Pas de réunion ultime du savoir. Savoir essentiellement classificateur; mais le "principe d'une classification ne se postule jamais. Seule l'enquête ethnographique peut le dégager a posteriori $»^{25}$. La pensée sauvage ne se donne pas des domaines parfaitement circonscrits en se situant en quelque sorte en face d'eux, mais elle met en relation des éléments qui ne sont en rien des entités existant en soi. Ces éléments n'existent que par les oppositions qu'ils entretiennent avec d'autres éléments et non dans une différence absolue. Le singulier, le différent ne le sont qu'en rapport à d'autres termes. Le réel n'est pas découpé, mais articulé. Tout ce qui est, pour elle, est immédiatement connu : «Tout offre un sens » et le concret ne saurait « receler un résidu d'inintelligibilité ${ }^{26}$. Le nouveau, l'évènement ont déjà leur place, au sens où ils ne pourraient la remettre en cause, l'amener à se retourner sur soi pour se reprendre ou se modifier dans sa démarche de connaissance. Pensée qui totalise, mais pensée qui n'accumule pas les connaissances, qui ne peut établir de bilan, dans ce sens, sans passé. L'idée d'un monde à connaître, d'un Inconnu 
n'a pas de sens. Pensée pour laquelle le réel n'est pas immédiatement opaque, donc à connaître, ce qui aurait pour conséquence que des zones entières resteraient dans l'ombre, de fait ou de droit. Ne se donnant pas comme confrontée au réel, elle n'a pas à envisager l'adéquation ou l'efficacité de ses procédures de connaissance : elle ne peut connaître le doute quant à ses moyens, ni envisager de se transformer dans son statut : elle est dans le monde.

«Son point de départ est sans importance [...] insoucieuse de partir ou d'aboutir franchement, la pensée mythique n'effectue pas de parcours entier : il lui reste toujours quelque chose à accomplir. Comme les rites, les mythes sont interminables. $»^{27}$

Cette pensée qui se développe en s'exposant, en s'extériorisant, n'est pas mue par la volonté de se rassembler, de reprendre ses productions, est en quelque sorte immédiate. Or le diacritisme instrumental de la pensée sauvage est proprement le moyen de cette prolifération des couples d'oppositions, de telle manière que cette pensée soit toujours inachevée et ne puisse coïncider avec soi, se retourner sur soi. L'exclusion d'une pensée-« sujet » irait de pair avec cette dichotomisation réitérée telle que la perte, la fuite en avant soient seules possibles. On voit donc, combien cette notion de Décision développée ici à propos de l'étude des sociétés sauvages par M. Gauchet, à la suite de $\mathrm{Cl}$. Lefort, est fondamentale et féconde, puisqu'elle permet de rendre compte du mode de fonctionnement de la pensée "sauvage", contre Levi-Strauss lui-même qui n'y décelait qu'un mode de fonctionnement de la pensée à l'état sauvage, cherchant même à fonder celui-ci dans l'ordre physico-chimique. Au contraire, si ce mode de fonctionnement de la pensée est institué (socialement) alors on doit pouvoir en rendre raison en le rapportant à une institution originelle.

\section{Le judaïsme antique, une société contre l'État?}

Deux interrogations pourraient provisoirement nous retenir. D'une part, qu'implique dans l'ordre de la Décision instauratrice du régime d'une société que la loi soit référée à un "sujet absolu», réconciliant en lui le savoir et la volonté? Qu'implique la transformation (nous ne supposons ici aucune continuité) de l'ailleurs, de l'avant, de l'autre d'une société, telle que nous est apparue la société sauvage, en un ailleurs, en un autre, en un avant, essentiellement caractérisés par un « sujet absolu »? D'autre part, si la société judaïque ne se pense qu'en référence à un "autre-sujet» absolu, cela implique-t-il nécessairement que, suivant le schéma plus haut exposé, elle puisse être caractérisée comme étant une société pour l'Etat, une société dans ce cas despotique ? Or, la société judaïque, sans tenir compte des périodes de l'Exil, n'a été que fort peu une société étatique, royale, puisque la royauté n'apparaît qu'avec Saül. En outre, il faudra tenir compte des réactions qui se sont toujours développées, tant au moment de l'émergence de la royauté, que lors des grandes crises sociales succédant à la dislocation de l'empire de Salomon, contre le pouvoir royal et la férocité des Grands. Nous avons donc affaire à une société qui théoriquement est théocratique et dont le caractère étatique est secondaire, advenu. S'il y a despotisme, celui-ci ne saurait la caractériser et par ailleurs il contribue à modifier les rapports des hommes à Dieu. Si la société judaïque est une société contre la médiation royale entre l'au-delà et l'ici-bas, il n'en reste pas moins que fondamentalement elle se pense en fonction de la médiation. Médiation originaire de Moïse, médiation continuée d'un corps de prêtres, des prophètes, puis des rabbins. Par ailleurs, il est peut-être de l'essence de toute 
médiation, et de celle-ci en particulier, de se donner comme inessentielle, ou transitoire : Israël, "royaume des prêtres" (Exode XIX, 6, 1) peut vouloir dire aussi bien qu'Israël est une théocratie, mais aussi qu'il n'y a aucun écart entre la loi et la société parce que la loi est devenue immanente à la société. De ce que l'autre est sujet absolu doit découler que la loi n'est plus immédiatement immanente à l'ordre social mais qu'elle doit le devenir dans une réconciliation qui sera aussi celle de Dieu et des hommes. Cet écart entre la loi qui devrait être immanente et sa situation de fait, c'està-dire son non-respect, l'existence continuelle du péché d'Israël, introduit la société dans l'historicité, dans le projet d'un devoir-être, d'une réunification. Tantôt, la médiation se pensera comme essentielle, et ce particulièrement dans les périodes de stabilité, tantôt, n'étant là qu'en vue de la réconciliation de l'ici-bas et de l'au-delà, se donnera comme circonstanciée. Tantôt le corps des prêtres affirmera sa filiation à la médiation originaire, à Moïse et se clamera héréditaire, tantôt des prophètes isolés manifesteront l'écart d'Israël à la loi, et l'urgence de la réconciliation. Nous avons postulé qu'on ne peut rendre compte du judaïsme antique qu'en faisant référence à une Décision instituante, mais elle-même ininstituée, de laquelle découle cette singulière mise en rapport du savoir, du pouvoir et de la loi qui caractérise cette société. C'est dire qu'on ne peut rendre compte par l'histoire, par l'événement, de ce régime spécifique, parce que lui-même institue l'histoire, fait que cette société se pense dans l'histoire, même si par ailleurs cette historicité est différente de la nôtre. Si l'on veut considérer autrement cette question, rappelons, et le problème est le même pour Clastres, que les sauvages n'avaient pas eu à faire l'expérience de l'oppression pour se vouloir contre l'Etat, mais que cette négation allait de pair avec une scission radicale de la société avec le lieu du haut duquel elle se pensait: un ensemble de décisions résultant d'une Décision instauratrice. Il serait ainsi tentant, pour rendre compte de cette société despotique sans despote d'en rechercher l'origine du côté d'une certaine articulation d'événements. En effet, pourquoi la société judaïque n'a-t-elle pas laissé émerger, entre elle et Dieu, une médiation royale, à la manière des sociétés voisines? Ou, lorsque celleci advint, pourquoi l'avoir laissée dans la précarité et le contingent? Les Hébreux ont pu vouloir se donner un maître absolu afin d'échapper aux petits maîtres royaux et en particulier aux petits rois des villes canaaéennes. En effet pour L. Caquot ${ }^{28}$, les Hébreux sont fondamentalement des exclus. Selon lui, il n'est pas invraisemblable qu'un groupe sémitique se soit installé pendant le nouvel empire en Egypte. Ces sémites auraient travaillé à la construction des grands monuments et certains auraient pu accéder à de hautes fonctions à la cour. La venue en Egypte de Jacob et ses fils n'est donc pas invraisemblable, non plus que la présence de Joseph et de Moïse à la cour du Pharaon. D'autre part, l'archéologie palestinienne révèle que des bouleversements ont eu lieu vers le milieu du -XIII è siècle, des villes anciennes ont été détruites, des sites abandonnés rebâtis. Il s'agit peut-être des traces de la conquête hébraïque de la Palestine. Pour lui, il ne faut pas séparer l'histoire biblique des Hébreux de ce qu'apprennent des textes du -XVè et du - XIV è sur les Khapirou. Selon les archives des villes d'Alalakh et d'Ougarit (Syrie) et la correspondance des cités-états de Palestine et de Syrie, adressée au Pharaon, il s'agirait de gens qui de toute manière sont en marge de la société urbaine: main d'œuvre temporaire, brigands, etc. Quelques textes égyptiens signalent des aperou, employés aux vendages. Les attestations du terme aperou/khapirou indiquent qu'il ne s'agit pas d'une ethnie, mais de la désignation d'une catégorie sociale. Il peut s'agir de déracinés, ayant dû fuir le territoire des cités. Par ailleurs, il n'est pas certain que le nom biblique des Hébreux ('ibrim) ait été à 
l'origine un nom ethnique. Plusieurs emplois de l'adjectif 'ibri pour qualifier un esclave (code de l'Alliance, Exode XXI,2), la valeur méprisante du terme dans la bouche des Philistins à I Samuel, IV, 6, 9, plaident en faveur d'un rapprochement entre 'ibri et khapirou. Les premiers Hébreux auraient été appelés ainsi en raison de leur déchéance sociale suggérée par la misère qui pousse Jacob en Egypte. Ce groupe a pu porter un temps le nom de Khapirou. D'autre part la conquête de la Palestine pourrait être la conséquence d'une révolution sociale: des couches inférieures de la population, victime de l'oppression des petits rois de Canaan, se seraient insurgées et auraient ruiné les villes royales. En se confédérant, ils auraient mis en commun leurs traditions et se seraient définis comme les descendants des mêmes ancêtres. Les souvenirs d'un séjour en Egypte seraient le bien propre d'un de ces groupes de conquérants. On admet une mise en commun des traditions, qui a peut-être permis aux légendes patriarcales de prendre place dans le patrimoine de tout Israël, alors qu'elles appartenaient à l'origine à des unités familiales ou locales. La communauté ne devait guère avoir le caractère fermé d'un groupe ethnique, mais était plutôt unie par une situation identique. Qu'il s'agisse d'anciens nomades ou de hors la loi, ils se trouvaient en butte à l'hostilité des Cité-Etats de Canaan dont la civilisation matérielle était très supérieure à la leur, d'où, selon Caquot, le refus du culte des images. Mais si nous les concevons comme l'histoire des khapirou s'agrégeant peu à peu au groupe des fuyards d'Egypte dirigés par Moïse, cimentant leur union par un lien religieux, devenant les maitres d'un vaste territoire en Palestine grâce à leur énergie, à une organisation de leur société plus juste que celle à laquelle ils voulaient échapper, et contraints de continuer la lutte contre la civilisation des villes et des empires, certains aspects fondamentaux de leur religion peuvent se trouver éclairés.

C'est dans cette perspective que L. Caquot interprète l'Alliance. Il note que la conception d'un dieu-maître de la collectivité humaine est généralement sémitique, ainsi que la supériorité d'un dieu sur celui des autres hommes. Seule l'alliance du dieu et des hommes serait proprement judaïque. Or cette alliance ne suppose pas d'égalité entre les partenaires. Il est vrai qu'Exode XIX. 4-6 indique que les rapports entre Israël et son dieu sont commandés par une sorte de réciprocité. De même le discours d'alliance que Josué XXIV met dans la bouche du successeur de Moïse lors de l'assemblée de Sichem: "Vous êtes témoins contre vous que vous avez choisi YWHW pour le servir » dit Josué au peuple après un long rappel des hauts faits de YHWH en faveur d'Israël. Et le Deutéronome attache la plus grande importance à la réciprocité des rapports en enseignant qu'Israël vivra s'il obéit à Dieu, sinon Dieu se retournera contre lui. Or, l'expression "conclure une alliance", (kârat berit: couper une alliance) apparaît au -15 è siècle sur une tablette cunéiforme de Qatna en Syrie du Nord. Et, par des témoignages hittites, on sait qu'un rituel d'alliance consistant à faire passer les contractants entre les deux moitiés d'un animal sacrifié, était largement répandu. Cet usage n'était pas étranger à Israël (alliance avec Abraham - Genèse XV -, Jérémie XXXIV,18). La tradition elohiste d'Exode XXIV 4,8 situe au pied de la montagne de Dieu une cérémonie durant laquelle Moïse asperge le peuple avec la moitié du sang d'une victime et un autel, représentant sans doute YHWH avec l'autre moitié. Ce rituel d'alliance avec la divinité est probablement l'adaptation d'un acte d'alliance entre partenaires humains. Par ailleurs, L. Caquot, note une ressemblance entre la structure littéraire des récits bibliques de conclusion et de renouvellement d'alliance avec Dieu et les traités hittites de vassalité. Ceux-ci présentent, en effet, un schéma assez rigoureux : on énonce la titulature de l'empereur hittite, on rappelle l'histoire de ses relations avec 
le vassal qui va sceller le traité, on énumère les conditions que doit remplir le vassal pour rester fidèle à l'alliance et conserver ainsi la protection de son suzerain, puis il est prescrit de déposer le texte du traité dans un temple et d'en donner lecture à l'occasion ; on mentionne les dieux invoqués comme témoins ; enfin viennent une série de malédictions et de bénédictions destinées au vassal selon qu'il violera ou respectera le traité. Or, on retrouve dans l'Exode, dans Josué XXIV et dans le Deutéronome divers éléments de ce schéma : les œuvres passées de YHWH, ses exigences, l'ordre de lire le livre de l'alliance, l'invocation de témoins, les malédictions et les bénédictions. Dieu est ainsi défini vis-à-vis d'Israël comme l'empereur hittite vis-à-vis de ses vassaux. De même certains éléments du schéma des traités de vassalité pourraient se retrouver plus tard dans les oracles prophétiques où YHWH intente un procès à son peuple. L'Alliance est au cœur des relations entre Israël et son dieu, elle a influencé les manifestations de la piété. La vertu religieuse par excellence est la khésèd, la loyauté qu'on doit à un allié. Si l'homme est fidèle à l'alliance, Dieu lui aussi est fidèle et ne cesse d'accorder sa protection. Mais la fidélité du partenaire divin est infiniment supérieure, alors que celle des hommes est constamment défaillante. Ainsi, la définition de Dieu comme un souverain qui s'engage par une alliance à protéger un partenaire inférieur en échange de la soumission de celui-ci à sa volonté a conféré une nouvelle valeur à la vieille conception sémitique du dieu-guide d'un groupe humain. YHWH est le "berger d'Israël" (Psaume XXIII, Genèse XLVIII.15, Ezéchiel XXXIV). Il en est aussi le roi. Quand les Hébreux offrent à Gédëon de devenir leur souverain, ce dernier refuse en déclarant : « je ne dominerai pas sur vous... C'est YHWH qui dominera sur vous » (Juges VIII,23). En conséquence aucune autorité humaine ne doit s'interposer entre Israël et son Dieu. C'est le sens de I Samuel VIII :

« Les anciens d'Israël demandèrent à Samuel d'établir sur eux un roi pour les juger, comme il y en a chez toutes les nations. Samuel vit avec déplaisir qu'ils disaient : donne-nous un Roi pour nous juger. Et Samuel pria l'Eternel. L'Eternel dit à Samuel: Ecoute la voix du peuple dans tout ce qu'il te dira; car ce n'est pas toi qu'ils rejettent, c'est moi qu'ils rejettent, afin que je ne règne plus sur eux. Ils agissent à ton égard comme ils ont toujours agi depuis que je les ai fait monter d'Egypte jusqu'à ce Jour; ils m'ont abandonné pour servir d'autres dieux. Ecoute donc leur voix ; mais donne leur des avertissements, et fais leur connaître le droit du roi qui régnera sur eux. »/Samuel dit au peuple/ « Voici quel sera le droit du roi qui régnera sur vous. Il prendra vos fils, et il les mettra sur ses chars et parmi ses cavaliers afin qu'ils courent devant son char; il s'en fera des chefs de mille et des chefs de cinquante, et il les emploiera à labourer ses terres, à récolter ses moissons, à fabriquer ses armes de guerre et l'attirail de ses chars. Il prendra vos filles pour en faire des prostituées, des cuisinières et des boulangères. Il prendra la meilleure partie de vos champs, de vos vignes et de vos oliviers, et la donnera à vos serviteurs. Il prendra la dîme du produit de vos semences et de vos vignes, et la donnera à ses serviteurs. Il prendra vos serviteurs et vos servantes, vos meilleurs bœufs et vos ânes, et s'en servira pour ses travaux. Il prendra la dîme de vos troupeaux, et vousmêmes serez ses esclaves. Et alors vous crierez contre votre roi que vous vous serez choisi, mais l'Eternel ne vous exaucera point. Le peuple refusa d'écouter la voix de Samuel...»

Texte capital. Mais il faudra déterminer si l'auteur de I Samuel refuse d'une manière radicale la médiation d'une autorité entre l'ici-bas et l'au-delà ou bien si son hostilité à la royauté est limitée à celle de Saül, la légitimité étant alors pour lui du côté de David et de sa lignée. Notons rapidement que l'émergence d'une royauté va de pair avec le renversement de la dette, avec l'asservissement des hommes et la constitution d'une armée de métier (cavalerie et chars) alors que l'armée des anciens Hébreux était celle 
de tout le peuple, son équipement était léger et sa tactique la guérilla. L. Caquot résume son interprétation de l'Alliance :

"la conviction religieuse que traduit le motif de l'Alliance avec un dieu se comprend par la situation des anciens Hébreux, en se plaçant par l'alliance sous l'autorité d'un dieu, ils se soustraient à celle des rois terrestres, du pharaon ou des petits-rois des cités-Etats cananéennes. Ils affirment leur complète indépendance et en même temps cimentent leur union, car les règles et les lois sans lesquelles aucune société ne peut vivre sont sanctionnées par le souverain divin en prenant la place des prescriptions du suzerain dans les traités de vassalité. $»^{29}$

A la suite de L. Caquot, on a l'impression que les Hébreux ont fait un certain nombre d'expériences, et en particulier celle de la servitude, que c'est à la suite d'une réflexion sur celles-ci qu'un "projet" de société aurait été élaboré, tel que la société aurait trouvé en un dieu-suzerain l'origine de sa loi. On voit la difficulté : en admettant qu'un peuple ou des tribus rassemblées aient pu s'instituer globalement à partir d'une réflexion commune sur des événements (internes ou externes -ce qui se passe ailleurs-), cela suppose que cette réflexion ait été possible, qu'il y ait eu un retour sur soi, qu'un sens ait été donné au passé ouvrant ainsi la possibilité d'un avenir. Or tout ceci suppose une Décision initiale : que la société puisse accueillir l'expérience et la reprendre, donc qu'il $\mathrm{y}$ ait déjà une place pour le nouveau, telle qu'il puisse faire problème. Mais nous savons que cette attitude même est instituée, qu'il ne va pas de soi qu'une société se penche sur son devenir.

Alors la narration de l'historien - ce récit des origines d'Israël essayant de fonder dans le temps ce qui va au contraire le faire être d'une certaine manière - n'est rien d'autre qu'un nouveau mythe des origines à ajouter à la liste déjà longue des traditions judaïques concernant l'origine d'Israël. Chercher dans une suite d'évènements l'origine d'une société, ou bien rendre compte de sa nature par la nature de ceux-ci, c'est toujours rester dans l'ordre de ce qu'elle dit d'elle-même, en restant dans la méconnaissance de son institution. La question ne se posera-t-elle pas alors pour toutes les caractéristiques de la religion? Suffira-t-il en effet d'expliquer cette pensée de l'émancipation et de la justice qu'est le judaïsme par une référence à la servitude supposée d'Israël en Egypte et sous le joug des royautés cananéennes? Ou par les effets de la crise sociale après la dislocation de l'empire de Salomon? Se pose la question du statut et de l'utilisation des textes de l'Ancien Testament, et ce en dehors même des questions posées par l'exégèse. On a vu que dans le cas de I Samuel VIII, l'interprétation dépendra déjà de ce que l'on sait de son auteur. Il est en effet probable que l'historien deutéronomiste prête ses intentions au héros qu'il met en scène. Ce "droit du roi" n'est pas une censure anticipée du despotisme de Salomon. Les documents de correspondance royale retrouvés à Ras Shamra ont montré que le droit du roi recouvre la pratique des roitelets de Syrie vers la fin du -II e millénaire. Il y aurait donc une base historique à la condamnation que Samuel porte contre la royauté. Mais par ailleurs nous savons que l'auteur de Samuel et des premiers livres des Rois, est tendancieux: après avoir abaissé Saül pour exalter David, il entend justifier par la volonté et les interventions de YHWH le principe de l'hérédité monarchique qui ne pouvait se recommander d'aucune tradition antérieure. Il veut aussi justifier l'avènement de Salomon, fait purement contingent. Ce texte donc, qui en apparence est une condamnation de la royauté "terrestre" pour restaurer les droits de la royauté divine, légitimerait une des formes historiques de la première. Est-ce dire qu'il n'était pas possible pour l'auteur de rendre compte d'une expérience - celle de l'asservissement - 
ayant eu lieu dans un territoire voisin, non parce qu'il était voisin, mais parce que cela supposerait un savoir de la société sur elle-même qu'elle ne saurait avoir? Est-il en effet possible que du sein même de la société hébraïque un savoir d'elle-même puisse éclore sans que celui-ci soit, profondément, méconnaissance de la Décision instauratrice?

\section{NOTES}

1. L'auteur et S. Voldman (On trouvera le témoignage de S. Voldman sur la Résistance juive en France, in Les juifs dans la résistance et la libération. Ed. du Scribe, 1985. J.L. Déotte est l'auteur d'une fresque de noms consacrée à l'extermination, in Le mémorial de la Paix, Caen.

2. Juifs et arabes, éd. Minuit, Paris, p. 104.

3. Un homme en trop, Seuil, Paris, 1976.

4. Les Sémites occidentaux. Les Arabes du Nord et du Centre, Histoire des Religions, T.1, La Pléïade.

5. Louis Caquot, opus cité.

6. Cl. Lefort : Esquisse d'une genèse de l'idéologie dans les sociétés modernes, in : Textures 8-9, 1974.

7. Cl. Lefort : La naissance de l'idéologie et l'humanisme, in : Textures, 6-7, 1973.

8. Marx, Principes d'une critique de l'économie politique (1857-1858), III. Formes précapitalistes de la production. Types de propriété, commune tribale et despotisme oriental, etc. In, CEuvres 2, Pléïade.

9. Cl. Lefort, opus cité : Esquisse....

10. P. Clastres : La société contre l'Etat, Minuit, p. 42.

11. Opus cité, p. 136.

12. J.P. Vernant : Espace et organisation politique en Grèce ancienne. Mythe et pensée chez les Grecs

13. Les maitres de vérité dans la Grèce archaïque, Maspéro, Paris.

14. Politique et société, Texture $n^{\circ} 10-11,1975$ et La leçon des sauvages, Texture $n^{\circ} 12-13,1975$.

15. Vocabulaire des institutions indo-européennes, vol 2, p. 20.

16. Cl. Lefort : L'échange et la lutte des hommes, Temps moderne, 1951.

17. Salhins : Politique de l'Essai sur le don.

18. J. Lizot: Le cercle des feux. Faits et dits des Indiens Yanomami, Le Seuil, p. 9.

19. «De la torture dans les sociétés primitives » in : La société contre l'Etat.

20. M. Gauchet, opus cité, p. 75.

21. H. Clastres, La Terre sans mal, Le Seuil, 1975

22. M. Gauchet, opus cité, p.88

23. Cl. Lefort et M. Gauchet, Sur la démocratie: le politique et l'institution du social, in: Textures $\mathrm{n}^{\circ} 2-3,1971$.

24. P. Clastres, La société contre l'Etat, p. 146.

25. Cl. Levi-Strauss, La pensée sauvage, Plon, p. 79.

26. Ibid., p. 228.

27. Levi-Strauss, Le Cru et le Cuit, Plon, p. 14.

28. L. Caquot, la religion d'Israël, L'histoire des religions, T.1, PLéiade.

29. Opus cité, p. 385. 


\section{RÉSUMÉS}

Ce texte de 1978 ne porte pas tant sur les origines historiques des royaumes d'Israël, auquel cas une réactualisation aurait été nécessaire en raison des dernières découvertes archéologiques et des hypothèses les plus récentes concernant la chronologie des textes de l'Ancien Testament, que sur la philosophie politique de $\mathrm{Cl}$. Lefort et de $\mathrm{P}$. Clastres que l'on cherche à mettre à l'épreuve à partir d'un "cas" significatif (le refus de l'instauration d'un roi sur Israël) relevant du théologicopoltique et du "despotisme oriental".

\section{INDEX}

Mots-clés : antiquité juive, Clastres (Pierre), événement, Lefort, société sauvage 